\title{
Enhanced critical temperature, pairing fluctuation effects, and BCS-BEC crossover in a two-band Fermi gas
}

\author{
Hiroyuki Tajima, ${ }^{1}$ Yuriy Yerin, ${ }^{2}$ Andrea Perali, ${ }^{3}$ and Pierbiagio Pieri ${ }^{2,4}$ \\ ${ }^{1}$ Quantum Hadron Physics Laboratory, RIKEN Nishina Center, Wako, Saitama 351-0198, Japan \\ ${ }^{2}$ School of Science and Technology, Physics Division, Università di Camerino, 62032 Camerino (MC), Italy \\ ${ }^{3}$ School of Pharmacy, Physics Unit, Università di Camerino, 62032 Camerino (MC), Italy \\ ${ }^{4}$ INFN, Sezione di Perugia, 06123 Perugia $(P G)$, Italy
}

(Received 31 January 2019; revised manuscript received 23 April 2019; published 28 May 2019)

\begin{abstract}
We study the superfluid critical temperature in a two-band attractive Fermi system with strong pairing fluctuations associated with both interband and intraband couplings. We focus specifically on a configuration where the intraband coupling is varied from weak to strong in a shallow band coupled to a weakly interacting deeper band. The whole crossover from the Bardeen-Cooper-Schrieffer (BCS) condensation of largely overlapping Cooper pairs to the Bose-Einstein condensation (BEC) of tightly bound molecules is covered by our analysis, which is based on the extension of the Nozières-Schmitt-Rink approach to a two-band system. In comparison with the single-band case, we find a strong enhancement of the critical temperature, a significant reduction of the preformed pair region where pseudogap effects are expected, and the entanglement of two kinds of composite bosons in the strong-coupling BEC regime.
\end{abstract}

DOI: 10.1103/PhysRevB.99.180503

Introduction. After the realization of the BCS-BEC crossover phenomenon in ultracold Fermi gases [1-3], which provided a unified understanding of both weak-coupling BCS Fermi superfluidity and Bose-Einstein condensation of molecular bosons [4-12], multicondensates can be regarded as the next paradigmatic systems to be explored. Owing to the emergence of additional degrees of freedom of the order parameter, these multicondensate systems can lead to a plethora of novel quantum phenomena. While even the single-component fermionic condensate has bridged various research fields such as nuclear physics [12-19] and several solid-state systems [4,7,8,20-31], the more generic concept of multicomponent BCS-BEC crossover not only builds up an interdisciplinary cross-link among strongly correlated systems but also opens a new frontier to explore the optimal configuration for high- $T_{\mathrm{c}}$ superconductivity $[32,33]$.

Among the variety of unconventional superconductors recently discovered, iron-based superconducting compounds are of particular interest due to their multiband electron structure with interband couplings producing complex order parameter symmetry and multiple energy gaps. This gives new opportunities to observe experimentally the BCSBEC crossover in a new class of superconducting materials [26-29,31,34-36]. Nanostructured superconductors are another promising class of materials in which the multiband BCS-BEC crossover, in the presence of shape resonance effects, can play a key role in the control and enhancement of superconductivity $[37,38]$. A multiband structure with a small Fermi surface pocket has an important role to achieve such strongly correlated crossover regime in all of these electron systems [39-42]. Some two-band theoretical models predict that so-called incipient bands may play an important role in several superconducting iron-based materials like FeSe intercalates and monolayers, considering them as quasi-twodimensional systems $[43,44]$. On the other hand, recent experiments indicate the presence of a three-dimensional (3D) momentum dependence of the gap in FeSe multiband superconductors, indicating that a 3D theoretical approach may be applied for the description of the superconducting state in this compound $[45,46]$.

A two-band Fermi system with Josephson-like interband coupling [47] is also under current experimental investigation in ultracold ${ }^{173} \mathrm{Yb}$ atomic Fermi gases near an orbital Feshbach resonance [48-50]. By applying a magnetic field, the energy separation between different atom-atom scattering channels (corresponding to that between two bands in the associated model Hamiltonian) can be arbitrarily tuned, and the emergence of the BCS-BEC crossover has been theoretically predicted [51-56]. In this case, however, the situation is complicated by the presence of additional deep molecular bound states, on top of the shallow one which is responsible for the orbital Feshbach resonance [53,56], which make the resulting model less relevant for the physics of multiband superconductors.

In this Rapid Communication, we address the effects of strong pairing fluctuations on the superfluid critical temperature for a two-band system with varying intra- and interband couplings. We focus in particular on the physically relevant configuration with a shallow "hot" band (in which the intraband coupling is varied from weak to strong) coupled with a deep "cold" band (with weak intraband coupling). For increasing hot-band coupling, we reveal a strong amplification of the critical temperature in comparison with the single-band case, with the interband coupling assisting such amplification, but not being crucial for its occurrence. In addition, in the intermediate (crossover) region between the BCS and 


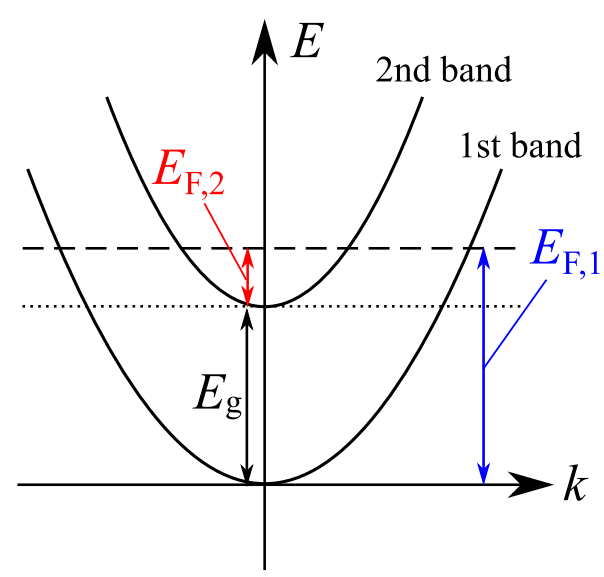

FIG. 1. The band structure considered in this work. The vertical and horizontal axes are the single-particle energy and momentum, respectively; $E_{g}$ is the energy shift between the first $(i=1)$ and the second $(i=2)$ band. $E_{\mathrm{F}, i}$ indicates the Fermi energy of $i$-band fermions in the absence of interactions.

Bose-Einstein condensation (BEC) limits, the comparison between the critical temperature and the pair-breaking temperature shows a significant shrinking of the preformed-pair region, implying a possible reduction of the pseudogap effects, in line with recent experimental findings for the FeSe multiband superconductors [57]. Finally, in the BEC regime with finite interband coupling, an interesting coherently coupled binary mixture of composite bosons is found.

Model. For the sake of generality, we consider the following minimal model Hamiltonian [58,59] for a threedimensional two-band Fermi system:

$$
H=\sum_{\boldsymbol{k}, \sigma, i} \xi_{\boldsymbol{k}, i} c_{\boldsymbol{k}, \sigma, i}^{\dagger} c_{\boldsymbol{k}, \sigma, i}+\sum_{i, j} U_{i j} \sum_{\boldsymbol{q}} b_{\boldsymbol{q}, i}^{\dagger} b_{\boldsymbol{q}, j} .
$$

Here, $c_{k, \sigma, i}$ is the annihilation operator of a fermion with spin $\sigma=\uparrow, \downarrow$ and band index $i=1,2, \quad b_{\boldsymbol{q}, i}^{\dagger}=$ $\sum_{k}^{k_{0}} c_{k+q / 2, \uparrow, i}^{\dagger} c_{-k+q / 2, \downarrow, i}^{\dagger}$ is the pair-creation operator in the $i$ band (where $k_{0}$ is a momentum cutoff), while $\xi_{k, 1}=k^{2} / 2 m-\mu, \quad \xi_{k, 2}=k^{2} / 2 m+E_{\mathrm{g}}-\mu$ are the kinetic energies measured from the chemical potential $\mu$ where $E_{\mathrm{g}}$ is the energy shift between the two bands, and $m$ is the particle (effective) mass (which is assumed to be identical in the two bands). We also introduce the band Fermi momenta $k_{\mathrm{F}, i}=\left(3 \pi^{2} n_{i}^{0}\right)^{1 / 3}$, defined in terms of the band densities $n_{1}^{0}$ and $n_{2}^{0}$ in the absence of any interactions and at zero temperature, with the corresponding Fermi energies $E_{\mathrm{F}, i}=k_{\mathrm{F}, i}^{2} / 2 m$ and temperatures $T_{\mathrm{F}, i}$, associated with these energies. In addition to that we will use the total Fermi momentum $k_{\mathrm{F}, \mathrm{t}}=\left(3 \pi^{2} n\right)^{1 / 3}$ defined by the total number density $n$, which is kept fixed. We set $k_{\mathrm{B}}, \hbar$, and the system volume $V$ equal to one. Figure 1 summarizes graphically our two-band configuration.

We express the intraband coupling $U_{i i}(<0)$ in terms of the intraband scattering length $a_{i i}[58,59]$ :

$$
\frac{m}{4 \pi a_{i i}}=\frac{1}{U_{i i}}+\sum_{k}^{k_{0}} \frac{m}{k^{2}} \text {. }
$$

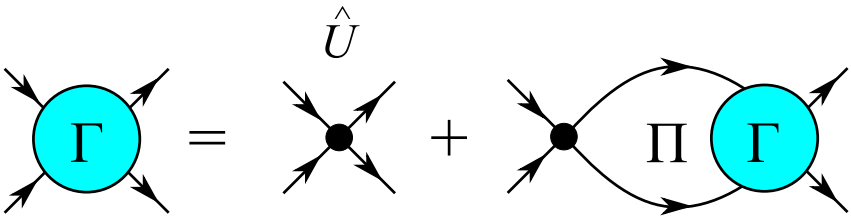

FIG. 2. Diagrammatic representation of the many-body $T$ matrix $\hat{\Gamma}$. $\hat{U}$ and $\hat{\Pi}$ are the $2 \times 2$ matrices of coupling constants and pair susceptibilities, respectively.

The momentum cutoff $k_{0}$ is considered to be much larger than the average interparticle distance, corresponding to a short-range condition on the interaction. Specifically, we take $k_{0}=100 k_{\mathrm{F}, \mathrm{t}}$. We choose the relatively large energy shift $E_{\mathrm{g}}=0.75 E_{\mathrm{F}, 1}=3 E_{\mathrm{F}, 2}$, which implies $k_{\mathrm{F}, 1}=(8 / 9)^{1 / 3} k_{\mathrm{F}, \mathrm{t}}$ and $k_{\mathrm{F}, 2}=(1 / 9)^{1 / 3} k_{\mathrm{F}, \mathrm{t}}$. We focus on the situation in which the shallow hot band $(i=2)$ undergoes the BCS-BEC crossover, whereas the intraband coupling in the deep cold band $(i=1)$ remains weak. In our configuration, this is achieved by taking $U_{22}=1.1 U_{11}$, which gives $\left(k_{\mathrm{F}, 1} a_{11}\right)^{-1}$ ranging between $\simeq-8$ and $\simeq-4$ when we change $\left(k_{\mathrm{F}, 2} a_{22}\right)^{-1}$ from the weakcoupling (BCS) to the strong-coupling (BEC) regime. We recall that for a single-band system the BCS-BEC crossover is driven by the dimensionless parameter $\left(k_{\mathrm{F}} a\right)^{-1}$, which ranges from $\left(k_{\mathrm{F}} a\right)^{-1} \lesssim-1$ in the weak-coupling (BCS) regime to $\left(k_{\mathrm{F}} a\right)^{-1} \gtrsim 1$ in the strong-coupling (BEC) regime.

The interband couplings are equal and real (guaranteeing the hermiticity of the Hamiltonian). For convenience, we introduce the dimensionless interband coupling $\tilde{U}_{12}$ by setting $U_{12}=\tilde{U}_{12}\left(k_{\mathrm{F}, \mathrm{t}} / k_{0}\right)^{2} E_{\mathrm{F}, \mathrm{t}} / n$, with $E_{\mathrm{F}, \mathrm{t}}=k_{\mathrm{F}, \mathrm{t}}^{2} / 2 m$. In this way, when $\tilde{U}_{12}$ ranges from 0 to 5 , the effects of the interband coupling on the quantities of interest will vary from weak to strong.

Formalism and results. The Nozières-Schmitt-Rink (NSR) formalism has been widely used for studying the BCS-BEC crossover in a single-band system. Reference [59] generalized the formalism to the two-band case, but only at the formal level. Here, we present an explicit numerical solution of the associated equations, and study the effect of pairing fluctuation at finite temperature in a two-band system across the whole BCS-BEC crossover.

Let us first briefly summarize the main equations of the NSR formalism for two-band systems. The sum of ladder diagrams defines the many-body $T$ matrix $\hat{\Gamma}$, as represented in Fig. 2, which satisfies

$$
\hat{\Gamma}\left(\boldsymbol{q}, i v_{l}\right)=\left[1+\hat{U} \hat{\Pi}\left(\boldsymbol{q}, i v_{l}\right)\right]^{-1} \hat{U},
$$

where $\hat{U}$ is the $2 \times 2$ matrix constructed with the interaction parameters $U_{i j}$, and

$$
\hat{\Pi}\left(\boldsymbol{q}, i v_{l}\right)=\left(\begin{array}{cc}
\Pi_{11}\left(\boldsymbol{q}, i v_{l}\right) & 0 \\
0 & \Pi_{22}\left(\boldsymbol{q}, i v_{l}\right)
\end{array}\right),
$$

where $v_{l}=2 l \pi T$ ( $l$ integer) is a boson Matsubara frequency at temperature $T$ and

$$
\Pi_{i i}\left(\boldsymbol{q}, i v_{l}\right)=T \sum_{\boldsymbol{k}, i \omega_{s}}^{k_{0}} G_{i}^{0}\left(\boldsymbol{q}-\boldsymbol{k}, i v_{l}-i \omega_{s}\right) G_{i}^{0}\left(\boldsymbol{k}, i \omega_{s}\right) .
$$




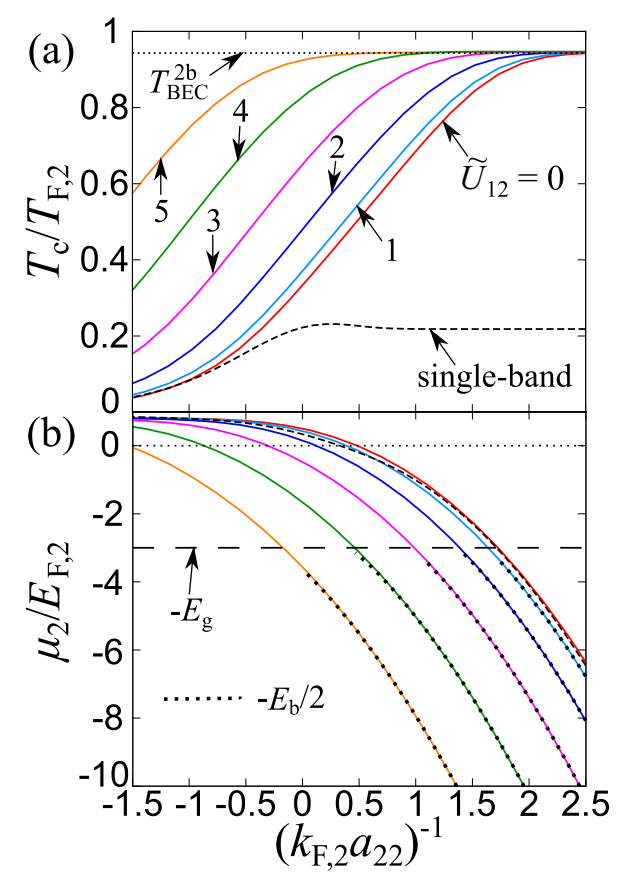

FIG. 3. (a) Critical temperature $T_{\mathrm{c}}$ and (b) chemical potential $\mu_{2} \equiv \mu-E_{\mathrm{g}}$ at $T_{c}$ vs $\left(k_{\mathrm{F}, 2} a_{22}\right)^{-1}$ at different interband couplings $\tilde{U}_{12}$ (we use the same line styles in both panels). The constant lines $T_{\mathrm{BEC}}^{2 \mathrm{~b}}=0.943 T_{\mathrm{F}, 2}$ and $-E_{\mathrm{g}}$, as well as the curves $-E_{\mathrm{b}} / 2$, with the two-body binding energy $E_{\mathrm{b}}$ obtained from Eq. (10) at different $\tilde{U}_{12}$ are also reported for reference.

Here $G_{i}^{0}\left(\boldsymbol{k}, i \omega_{s}\right)=1 /\left(i \omega_{s}-\xi_{\boldsymbol{k}, i}\right)$ is the bare Green's function of a $i$-band particle and $\omega_{s}=(2 s+1) \pi T$ ( $s$ integer) is a fermion Matsubara frequency.

The critical temperature $T_{\mathrm{c}}$ is determined by the Thouless criterion [60], namely, the divergence of $\hat{\Gamma}(0,0)$, corresponding to the condition

$$
\operatorname{det}\left[1+\hat{U} \hat{\Pi}\left(\boldsymbol{q}=0, i v_{l}=0\right)\right]=0 .
$$

This equation needs to be solved together with the particle number equation $n=-\partial \Omega / \partial \mu$, where $\Omega$ is obtained by adding the thermodynamic potential constructed from the ladder diagrams to the free one [6,59]. It is easy to show that in the present two-band case, $-\partial \Omega / \partial \mu$ can be expressed as the sum $n_{1}+n_{2}$ of the densities in the two bands, with

$$
n_{i}=2\left[\sum_{\boldsymbol{k}} f\left(\xi_{\boldsymbol{k}, i}\right)+T \sum_{\boldsymbol{k}, i \omega_{s}}^{k_{0}} G_{i}^{0}\left(\boldsymbol{k}, i \omega_{s}\right)^{2} \Sigma_{i}\left(\boldsymbol{k}, i \omega_{s}\right)\right],
$$

where $f$ is the Fermi function at temperature $T$ and we have introduced the self-energy

$$
\Sigma_{i}\left(\boldsymbol{k}, i \omega_{s}\right)=T \sum_{\boldsymbol{q}, i v_{l}} \Gamma_{i i}\left(\boldsymbol{q}, i v_{l}\right) G_{i}^{0}\left(\boldsymbol{q}-\boldsymbol{k}, i v_{l}-i \omega_{s}\right)
$$

for fermions in band $i$. At fixed $n, T$, and interaction parameters, the inversion of the number equation $n=n_{1}(\mu)+$ $n_{2}(\mu)$ determines the chemical potential $\mu$. Figure 3 shows the overall critical temperature $T_{\mathrm{c}}$ and the corresponding chemical potential $\mu-E_{\mathrm{g}} \equiv \mu_{2}$ measured from the bottom of the hot band as a function of $\left(k_{\mathrm{F}, 2} a_{22}\right)^{-1}$. We compare the results of the two-band system with the case in which the hot band is considered as a single band, with density fixed to $n_{2}^{0}$ (for our choice of $E_{\mathrm{F}, i}, n_{2}^{0}=n / 9$ ). Note that the single-band case differs from simply setting $U_{12}=0$ in the two-band system, for which a particle transfer between the two bands is possible. One sees indeed that for vanishing interband interaction, while in the weak-coupling (BCS) regime $T_{\mathrm{c}}$ and $\mu_{2}$ essentially coincide with the corresponding single-band results, in the intermediate and strong-coupling regions, $T_{\mathrm{c}}$ is greatly enhanced in comparison with the singleband case. In the two-band system, the pairing attraction in the hot band drains particles from the cold band to lower the overall free energy. The critical temperature is then enhanced, until in the strong-coupling limit the asymptotic value $T_{\mathrm{BEC}}^{2 \mathrm{~b}}=0.218\left(k_{\mathrm{F}, \mathrm{t}} / k_{\mathrm{F}, 2}\right)^{2} T_{\mathrm{F}, 2}=0.943 T_{\mathrm{F}, 2}$ is reached, corresponding to the condensation temperature for a gas of noninteracting bosons of density $n / 2$ and mass $2 m$. In this limit, $\mu_{2}$ coincides with $-E_{\mathrm{b}} / 2$, where $E_{\mathrm{b}}$ is the two-body binding energy, which for the decoupled system is given by $E_{\mathrm{b}}=$ $\left(m a_{22}^{2}\right)^{-1}$.

Even more interesting is the situation with a finite interband coupling. In this case the BEC limit can be effectively reached even for rather weak values of the intraband coupling $\left(k_{\mathrm{F}, 2} a_{22}\right)^{-1}$. This behavior can be understood by noting that the Thouless criterion (6) can be rewritten as $1+U_{\text {eff }} \Pi_{22}(0,0)=0$, where

$$
U_{\text {eff }}=U_{22}-U_{12}^{2} \Pi_{11}(0,0) /\left[1+U_{11} \Pi_{11}(0,0)\right],
$$

is the effective interaction determining $T_{\mathrm{c}}$. It is the second term in Eq. (9) which leads to a significant increase of the effective pairing interaction when $U_{12}$ increases. It can be shown in particular that in our configuration this term effectively corresponds to a shift $\simeq-0.5 k_{\mathrm{F}, 1} a_{11} \tilde{U}_{12}^{2}$ of the dimensionless coupling $\left(k_{\mathrm{F}, 2} a_{22}\right)^{-1}$ toward stronger couplings. The effect of the interband coupling can also be seen at the two-body level on the binding energy $E_{\mathrm{b}}$, which for the coupled system can be obtained from the equation determining the pole of the two-body $T$ operator on the real energy axis

$$
\left(1+U_{11} \Pi_{11}^{0}\right)\left(1+U_{22} \Pi_{22}^{0}\right)-U_{12}^{2} \Pi_{11}^{0} \Pi_{22}^{0}=0,
$$

where the vacuum particle-particle bubbles $\Pi_{i i}^{0}$ are obtained from $\Pi_{i i}\left(\boldsymbol{q}, i v_{l}\right)$ by setting $\boldsymbol{q}=0, i v_{l} \rightarrow-\left(E_{\mathrm{b}}-2 E_{\mathrm{g}}\right)-2 \mu$ and then taking the vacuum limit $\mu / T \rightarrow-\infty$. One then gets from Eq. (5)

$$
\Pi_{i i}^{0}=\frac{m k_{0}}{2 \pi^{2}}\left[1-\frac{\sqrt{m\left|E_{i}\right|}}{k_{0}} \arctan \left(\frac{k_{0}}{\sqrt{m\left|E_{i}\right|}}\right)\right],
$$

where we have defined $E_{i} \equiv-E_{\mathrm{b}}+2 E_{\mathrm{g}} \delta_{i, 1}$. Note that the binding energy $E_{\mathrm{b}}$ refers to the bottom of the upper band, and $E_{\mathrm{b}}>2 E_{\mathrm{g}}$ for $U_{12} \neq 0$. One sees in Fig. 3(b) that when $\tilde{U}_{12}$ increases, the binding energy $E_{\mathrm{b}}$ also increases, and $\mu_{2}$ approaches the BEC limit $-E_{\mathrm{b}} / 2$ at progressively weaker intraband couplings $\left(k_{\mathrm{F}, 2} a_{22}\right)^{-1}$.

Figure 4 focuses on the behavior of $\mu_{2}$ near the unitarity limit $\left(k_{\mathrm{F}, 2} a_{22}\right)^{-1}=0$. One can see that in the absence or for weak interband coupling, the chemical potential for the two-band system is larger than for a single band, such that the crossing of the bottom of the upper band is shifted to larger values of $\left(k_{\mathrm{F}, 2} a_{22}\right)^{-1}=0$. Physically, this is explained by the Pauli-blocking effect due to the occupied states in the cold 


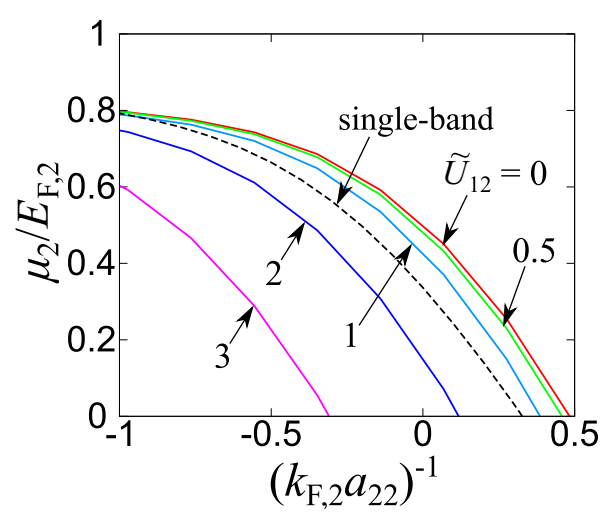

FIG. 4. Chemical potential $\mu_{2} \equiv \mu-E_{\mathrm{g}}$ vs $\left(k_{\mathrm{F}, 2} a_{22}\right)^{-1}$ at $T=T_{\mathrm{c}}$ near the unitarity limit $\left(k_{\mathrm{F}, 2} a_{22}\right)^{-1}=0$.

band which acts to retard the "bosonization" of the Cooper pairs. However, when the interband coupling increases, it overcomes this quantum statistical effect and eventually shifts the bosonization to smaller intraband coupling values. Figure 5 shows how the fermions distribute between the two bands when the coupling $\left(k_{\mathrm{F}, 2} a_{22}\right)^{-1}$ is varied. For different values of the interband coupling $\tilde{U}_{12}$, one observes in general a progressive transfer of particles from the cold band to the hot one as $\left(k_{\mathrm{F}, 2} a_{22}\right)^{-1}$ increases. However, while in the BEC limit one has a full transfer for $\tilde{U}_{12}=0$, at finite interband coupling the transfer is only partial. This behavior can be understood by solving the two-body bound-state equation $H_{\text {rel }} \Psi=E \Psi$ for the relative motion wave function $\Psi(r)=\left(\psi_{1}(r), \psi_{2}(r)\right)$, where $\psi_{1}$ and $\psi_{2}$ are the components in the two bands, $H_{\text {rel }}$ is the two-body relative motion Hamiltonian associated with the many-body Hamiltonian (1), and $r$ is the relative distance. In this way one finds that when $\tilde{U}_{12} \neq 0$ the bound-state solution has components in both bands, and the asymptotic value of $n_{i} / n$ in the BEC limit of the many-body problem coincides with the ratio $\left\|\psi_{i}\right\|^{2} /\left(\left\|\psi_{1}\right\|^{2}+\left\|\psi_{2}\right\|^{2}\right)$ in the two-body problem (dotted lines in Fig. 5). Here, $\left\|\psi_{i}\right\|^{2}=\int d^{3} r\left|\psi_{i}(r)\right|^{2}$; note that the condition $E_{\mathrm{b}}>2 E_{\mathrm{g}}$ is required to have $\left\|\psi_{1}\right\|<\infty$.

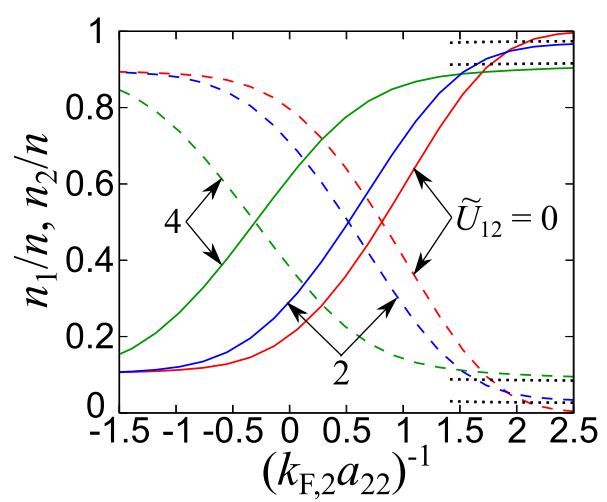

FIG. 5. Number densities $n_{i}$ (in units of $\left.n\right)$ in the lower $(i=1$, dashed line) and upper ( $i=2$, solid line) bands at $T_{\mathrm{c}}$ vs $\left(k_{\mathrm{F}, 2} a_{22}\right)^{-1}$ for different values of $\tilde{U}_{12}$. The dotted lines correspond to the ratios $\left\|\psi_{i}\right\|^{2} /\left(\left\|\psi_{1}\right\|^{2}+\left\|\psi_{2}\right\|^{2}\right)$ in the two-body problem (see text) for $i=1, \tilde{U}_{12}=2,4$ and $i=2, \tilde{U}_{12}=4,2$, from bottom to top.

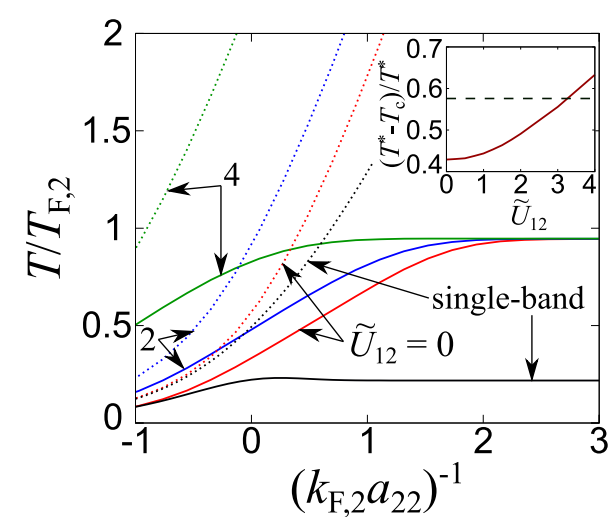

FIG. 6. Critical temperature $T_{\mathrm{c}}$ (solid lines) and pair-breaking temperature $T^{*}$ (dotted lines) as estimated by the mean-field critical temperature, vs $\left(k_{\mathrm{F}, 2} a_{22}\right)^{-1}$ for different values of $\tilde{U}_{12}$. The inset shows the ratio $\left(T^{*}-T_{\mathrm{c}}\right) / T^{*}$ near unitarity $\left[\left(k_{\mathrm{F}, 2} a_{22}\right)^{-1}=0.07\right]$ as a function of $\tilde{U}_{12}$. The corresponding value for the single-band case is also reported (dashed line).

In this extreme BEC regime, the bosons condensing at $T_{\mathrm{c}}$ are a coherent superposition of two molecular states in the two bands, with wave functions $\psi_{i}(r) \propto e^{-\sqrt{m\left|E_{i}\right| r}} / r$ and sizes $R_{1}=1 / \sqrt{m\left|2 E_{\mathrm{g}}-E_{\mathrm{b}}\right|}$ and $R_{2}=1 / \sqrt{m\left|E_{\mathrm{b}}\right|}$. In the less extreme regime whereby the chemical potential $\mu_{2}$ is between the bottom of the two bands, the two-body bound state is effectively replaced by a quasibound state, i.e., a resonance whose energy $E_{\mathrm{r}}$ essentially determines the value of the chemical potential $\left(\mu_{2} \simeq E_{\mathrm{r}} / 2\right)$. The energy $E_{\mathrm{r}}$ corresponds to a peak (narrow for small $\tilde{U}_{12}$ ) of the two-body $T$ matrix, while only the solution of the many-body problem yields the relative distribution of particles between the two bands. Interestingly, unusual vortex configurations with nontriangular geometry, stripes, or multiquantum-vortex lattices are expected to occur in two-band superconductors with nonequal pair sizes $[41,61]$ and two-species BEC $[62,63]$.

In Fig. 6 the preformed pair region of the phase diagram between the mean-field temperature $T^{*}$ and the superfluid critical temperature $T_{\mathrm{c}}$ is investigated for different interband coupling $\tilde{U}_{12}$. This region is of particular interest because below $T^{*}$, pseudogap phenomena and molecularlike pairing are expected to appear, with detectable signatures in the single-particle excitation spectra, depending on the intraband coupling strength $[64,65]$. Also, the amount of pair fluctuations in this region is responsible for the detrimental suppression of the superfluid critical temperature. The pairing temperature $T^{*}$ is strongly enhanced by increasing $\tilde{U}_{12}$. It is already larger than the corresponding temperature for the single-band case for vanishing interband coupling, because of the larger chemical potential (see Fig. 4). On the other hand, for intraband couplings in the hot band close to unitarity and in a sizable range of $\tilde{U}_{12} \lesssim 3$, the preformed pair region is reduced with respect to the single-band case, as quantified by the temperature window $\left(T^{*}-T_{\mathrm{c}}\right) / T^{*}$, reported in the inset of Fig. 6. This can be connected with the recent experiment in the multiband FeSe superconductor where BCS-BEC crossover signatures have been confirmed while a pseudogap was not detected [57]. 
Acknowledgments. H.T. thanks C. A. R. Sá de Melo for useful discussions and acknowledges the hospitality of the Physics Division at the University of Camerino, where most of this work was done. H.T. also thanks Y. Kondo for discussions. H.T. was supported by a Grant-in-Aid for JSPS fellows (Grant No. 17J03975). This work was supported by the Italian MIUR through the PRIN 2015 program (Contract No. 2015C5SEJJ001) and by the RIKEN iTHEMS program.
[1] C. A. Regal, M. Greiner, and D. S. Jin, Observation of Resonance Condensation of Fermionic Atom Pairs, Phys. Rev. Lett. 92, 040403 (2004).

[2] M. Bartenstein, A. Altmeyer, S. Riedl, S. Jochim, C. Chin, J. Hecker Denschlag, and R. Grimm, Crossover from a Molecular Bose-Einstein Condensate to a Degenerate Fermi Gas, Phys. Rev. Lett. 92, 120401 (2004).

[3] M. W. Zwierlein, C. A. Stan, C. H. Schunk, S. M. F. Raupach, A. J. Kerman, and W. Ketterle, Condensation of Pairs of Fermionic Atoms near a Feshbach Resonance, Phys. Rev. Lett. 92, 120403 (2004).

[4] D. M. Eagles, Possible pairing without superconductivity at low carrier concentrations in bulk and thin-film superconducting semiconductors, Phys. Rev. 186, 456 (1969).

[5] A. J. Leggett, Diatomic molecules and Cooper pairs, in Modern Trends in the Theory of Condensed Matter, edited by A. Peralski and R. Przystawa (Springer-Verlag, Berlin, 1980).

[6] P. Nozières and S. Schmitt-Rink, Bose condensation in an attractive fermion gas: From weak to strong coupling superconductivity, J. Low Temp. Phys. 59, 195 (1985).

[7] C. A. R. Sá de Melo, M. Randeria, and J. R. Engelbrecht, Crossover from BCS to Bose Superconductivity: Transition Temperature and Time-Dependent Ginzburg-Landau Theory, Phys. Rev. Lett. 71, 3202 (1993).

[8] A. Perali, P. Pieri, G. C. Strinati, and C. Castellani, Pseudogap and spectral function from superconducting fluctuations to the bosonic limit, Phys. Rev. B 66, 024510 (2002).

[9] Y. Ohashi and A. Griffin, BCS-BEC Crossover in a Gas of Fermi Atoms with a Feshbach Resonance, Phys. Rev. Lett. 89, 130402 (2002).

[10] S. Giorgini, L. P. Pitaevskii, and S. Stringari, Theory of ultracold atomic Fermi gases, Rev. Mod. Phys. 80, 1215 (2008).

[11] I. Bloch, J. Dalibard, and W. Zwerger, Many-body physics with ultracold gases, Rev. Mod. Phys. 80, 885 (2008).

[12] G. C. Strinati, P. Pieri, G. Röpke, P. Schuck, and M. Urban, The BCS-BEC crossover: From ultra-cold Fermi gases to nuclear systems, Phys. Rep. 738, 1 (2018).

[13] M. Baldo, U. Lombardo, and P. Schuck, Deuteron formation in expanding nuclear matter from a strong coupling BCS approach, Phys. Rev. C 52, 975 (1995).

[14] H. Stein, A. Schnell, T. Alm, and G. Röpke, Correlations and pairing in nuclear matter within the Nozières-Schmitt-Rink approach, Z. Phys. A 351, 295 (1995).

[15] U. Lombardo, P. Nozières, P. Schuck, H.-J. Schulze, and A. Sedrakian, Transition from BCS pairing to Bose-Einstein condensation in low-density asymmetric nuclear matter, Phys. Rev. C 64, 064314 (2001).

[16] A. Gezerlis and J. Carlson, Strongly paired fermions: Cold atoms and neutron matter, Phys. Rev. C 77, 032801(R) (2008).

[17] M. Jin, M. Urban, and P. Schuck, BEC-BCS crossover and the liquid-gas phase transition in hot and dense nuclear matter, Phys. Rev. C 82, 024911 (2010).
[18] S. Ramanan and M. Urban, BEC-BCS crossover in neutron matter with renormalization-group-based effective interaction, Phys. Rev. C 88, 054315 (2013).

[19] P. van Wyk, H. Tajima, D. Inotani, A. Ohnishi, and Y. Ohashi, Superfluid Fermi atomic gas as a quantum simulator for the study of the neutron-star equation of state in the low density region, Phys. Rev. A 97, 013601 (2018).

[20] R. Micnas, J. Ranninger, and S. Robaszkiewicz, Superconductivity in narrow-band systems with local nonretarded attractive interactions, Rev. Mod. Phys. 62, 113 (1990).

[21] M. Randeria, N. Trivedi, A. Moreo, and R. T. Scalettar, Pairing and Spin Gap in the Normal State of Short Coherence Length Superconductors, Phys. Rev. Lett. 69, 2001 (1992).

[22] J. Maly, K. Levin, and D. Z. Liu, Coulomb correlations and pseudogap effects in a preformed pair model for the cuprates, Phys. Rev. B 54, R15657(R) (1996).

[23] Y. Tomio, K. Honda, and T. Ogawa, Excitonic BCS-BEC crossover at finite temperature: Effects of repulsion and electron-hole mass difference, Phys. Rev. B 73, 235108 (2006).

[24] P. Pieri, D. Neilson, and G. C. Strinati, Effects of density imbalance on the BCS-BEC crossover in semiconductor electronhole bilayers, Phys. Rev. B 75, 113301 (2007).

[25] Y. Chen, A. A. Shanenko, A. Perali, and F. M. Peeters, Superconducting nanofilms: molecule-like pairing induced by quantum confinement, J. Phys.: Condens. Matter 24, 185701 (2012).

[26] Y. Lubashevsky, E. Lahoud, K. Chashka, D. Podolsky, and A. Kanigel, Shallow pockets and very strong coupling superconductivity in $\mathrm{FeSe}_{x} \mathrm{Te}_{1-x}$, Nat. Phys. 8, 309 (2012).

[27] A. Bianconi, Shape resonances in superstripes, Nat. Phys. 9, 536 (2013).

[28] K. Okazaki, Y. Ito, Y. Ota, Y. Kotani, T. Shimojima, T. Kiss, S. Watanabe, C.-T. Chen, S. Niitaka, T. Hanaguri, H. Takagi, A. Chainani, and S. Shin, Superconductivity in an electron band just above the Fermi level: Possible route to BCS-BEC superconductivity, Sci. Rep. 4, 4109 (2014).

[29] S. Kasahara, T. Watashige, T. Hanaguri, Y. Kohsaka, T. Yamashita, Y. Shimoyama, Y. Mizukami, R. Endo, and H. Ikeda, Field-induced superconducting phase of FeSe in the BCS-BEC cross-over, Proc. Natl. Acad. Sci. USA 111, 16309 (2014).

[30] S. Conti, A. Perali, F. M. Peeters, and D. Neilson, Multicomponent Electron-Hole Superfluidity and the BCS-BEC Crossover in Double Bilayer Graphene, Phys. Rev. Lett. 119, 257002 (2017).

[31] S. Kasahara, T. Yamashita, A. Shi, R. Kobayashi, Y. Shimoyama, T. Watashige, K. Ishida, T. Terashima, T. Wolf, F. Hardy, C. Meingast, H. v. Löhneysen, A. Levchenko, T. Shibauchi, and Y. Matsuda, Giant superconducting fluctuations in the compensated semimetal FeSe at the BCS-BEC crossover, Nat. Commun. 7, 12843 (2016).

[32] M. V. Milošević and A. Perali, Emergent phenomena in multicomponent superconductivity: An introduction to the focus issue, Supercond. Sci. Technol. 28, 060201 (2015). 
[33] D. Innocenti, N. Poccia, A. Ricci, A. Valletta, S. Caprara, A. Perali, and A. Bianconi, Resonant and cross-over phenomena in a multiband superconductor: Tuning the chemical potential near a band edge, Phys. Rev. B 82, 184528 (2010).

[34] K. Hashimoto, K. Cho, T. Shibauchi, S. Kasahara, Y. Mizukami, R. Katsumata, Y. Tsuruhara, T. Terashima, H. Ikeda, M. A. Tanatar, H. Kitano, N. Salovich, R. W. Giannetta, P. Walmsley, A. Carrington, R. Prozorov, and Y. Matsuda, A sharp peak of the zero-temperature penetration depth at optimal composition in $\mathrm{BaFe}_{2}\left(\mathrm{As}_{1-x} \mathrm{P}_{x}\right)_{2}$, Science 336, 1554 (2012).

[35] T. Shibauchi, A. Carrington, and Y. Matsuda, A quantum critical point lying beneath the superconducting dome in iron pnictides, Annu. Rev. Condens. Matter Phys. 5, 113 (2014).

[36] S. Rinott, K. B. Chashka, A. Ribak, E. D. L. Rienks, A. TalebIbrahimi, P. Le Fevre, F. Bertran, M. Randeria, and A. Kanigel, Tuning across the BCS-BEC crossover in the multiband superconductor $\mathrm{Fe}_{1+y} \mathrm{Se}_{x} \mathrm{Te}_{1-x}$ : An angle-resolved photoemission study, Sci. Adv. 3, 1602372 (2017).

[37] A. Perali, A. Bianconi, A. Lanzara, and N. L. Saini, The gap amplification at a shape resonance in a superlattice of quantum stripes: A mechanism for high $\mathcal{T}_{c}$, Solid State Commun. 100, 181 (1996).

[38] A. Bianconi, A. Valletta, A. Perali, and N. L. Saini, Superconductivity of a striped phase at the atomic limit, Physica C 296, 269 (1998).

[39] A. Guidini and A. Perali, Band-edge BCS-BEC crossover in a two-band superconductor: Physical properties and detection parameters, Supercond. Sci. Technol. 27, 124002 (2014).

[40] A. V. Chubukov, I. Eremin, and D. V. Efremov, Superconductivity vs bound state formation in a two-band superconductor with small Fermi energy: Applications to Fe-pnictides/chalcogenides and doped $\mathrm{SrTiO}_{3}$, Phys. Rev. B 93, 174516 (2016).

[41] S. Wolf, A. Vagov, A. A. Shanenko, V. M. Axt, A. Perali, and J. Albino Aguiar, BCS-BEC crossover induced by a shallow band: Pushing standard superconductivity types apart, Phys. Rev. B 95, 094521 (2017).

[42] L. Salasnich, A. A. Shanenko, A. Vagov, J. Albino Aguiar, and A. Perali, Screening of pair fluctuations in superconductors with coupled shallow and deep bands: A route to higher temperature superconductivity, arXiv:1810.03321.

[43] X. Chen, S. Maiti, A. Linscheid, and P. J. Hirschfeld, Electron pairing in the presence of incipient bands in iron-based superconductors, Phys. Rev. B 92, 224514 (2015).

[44] A. Linscheid, S. Maiti, Y. Wang, S. Johnston, and P. J. Hirschfeld, High $T_{\mathrm{c}}$ via Spin Fluctuations from Incipient Bands: Application to Monolayers and Intercalates of FeSe, Phys. Rev. Lett. 117, 077003 (2016).

[45] J. Ge, S. Cao, S. Yuan, B. Kang, and J. Zhang, Threedimensional superconductivity in nominal composition $\mathrm{Fe}_{1.03} \mathrm{Se}$ with $T_{\mathrm{c}}^{\text {zero }}$ up to $10.9 \mathrm{~K}$ induced by internal strain, J. Appl. Phys. 108, 053903 (2010).

[46] Y. S. Kushnirenko, A. V. Fedorov, E. Haubold, S. Thirupathaiah, T. Wolf, S. Aswartham, I. Morozov, T. K. Kim, B. Büchner, and S. V. Borisenko, Three-dimensional superconducting gap in FeSe from angle-resolved photoemission spectroscopy, Phys. Rev. B 97, 180501(R) (2018).

[47] H. Suhl, B. T. Matthias, and L. R. Walker, Bardeen-CooperSchrieffer Theory of Superconductivity in the Case of Overlapping Bands, Phys. Rev. Lett. 3, 552 (1959).
[48] R. Zhang, Y. Cheng, H. Zhai, and P. Zhang, Orbital Feshbach Resonance in Alkali-Earth Atoms, Phys. Rev. Lett. 115, 135301 (2015).

[49] G. Pagano, M. Mancini, G. Cappellini, L. Livi, C. Sias, J. Catani, M. Inguscio, and L. Fallani, Strongly Interacting Gas of Two-Electron Fermions at an Orbital Feshbach Resonance, Phys. Rev. Lett. 115, 265301 (2015).

[50] M. Höfer, L. Riegger, F. Scazza, C. Hofrichter, D. R. Fernandes, M. M. Parish, J. Levinsen, I. Bloch, and S. Fölling, Observation of an Orbital Interaction-Induced Feshbach Resonance in ${ }^{173} \mathrm{Yb}$, Phys. Rev. Lett. 115, 265302 (2015).

[51] M. Iskin, Two-band superfluidity and intrinsic Josephson effect in alkaline-earth-metal Fermi gases across an orbital Feshbach resonance, Phys. Rev. A 94, 011604(R) (2016).

[52] L. He, J. Wang, S.-G. Peng, X.-J. Liu, and H. Hu, Strongly correlated Fermi superfluid near an orbital Feshbach resonance: Stability, equation of state, and Leggett mode, Phys. Rev. A 94, 043624 (2016).

[53] J. Xu, R. Zhang, Y. Cheng, P. Zhang, R. Qi, and H. Zhai, Reaching a Fermi-superfluid state near an orbital Feshbach resonance, Phys. Rev. A 94, 033609 (2016).

[54] M. Iskin, Trapped ${ }^{173} \mathrm{Yb}$ Fermi gas across an orbital Feshbach resonance, Phys. Rev. A 95, 013618 (2017).

[55] P. Zou, L. He, X.-J. Liu, and H. Hu, Strongly interacting Sarma superfluid near orbital Feshbach resonance, Phys. Rev. A 97, 043616 (2018).

[56] S. Mondal, D. Inotani, and Y. Ohashi, Single-particle excitations and strong coupling effects in the BCS-BEC crossover regime of a rare-earth Fermi gas with an orbital Feshbach resonance, J. Phys. Soc. Jpn. 87, 084302 (2018).

[57] T. Hanaguri, S. Kasahara, J. Böker, I. Eremin, T. Shibauchi, and Y. Matsuda, Quantum Vortex Core and Missing Pseudogap in the Multiband BCS-BEC Crossover Superconductor FeSe, Phys. Rev. Lett. 122, 077001 (2019).

[58] M. Iskin and C. A. R. Sá de Melo, BCS-BEC crossover of collective excitations in two-band superfluids, Phys. Rev. B 72, 024512 (2005).

[59] M. Iskin and C. A. R. Sá de Melo, Two-band superfluidity from the BCS to the BEC limit, Phys. Rev. B 74, 144517 (2006).

[60] D. J. Thouless, Perturbation theory in statistical mechanics and the theory of superconductivity, Ann. Phys. (NY) 10, 553 (1960).

[61] E. Babaev and M. Speight, Semi-Meissner state and neither type-I nor type-II superconductivity in multicomponent superconductors, Phys. Rev. B 72, 180502(R) (2005).

[62] E. J. Mueller and Tin-Lun Ho, Two-Component Bose-Einstein Condensates with a Large Number of Vortices, Phys. Rev. Lett. 88, 180403 (2002).

[63] P. Kuopanportti, J. A. M. Huhtamäki, and M. Möttönen, Exotic vortex lattices in two-species Bose-Einstein condensates, Phys. Rev. A 85, 043613 (2012).

[64] J. P. Gaebler, J. T. Stewart, T. E. Drake, D. S. Jin, A. Perali, P. Pieri, and G. C. Strinati, Observation of pseudogap behaviour in a strongly interacting Fermi gas, Nat. Phys. 6, 569 (2010).

[65] A. Perali, F. Palestini, P. Pieri, G. C. Strinati, J. T. Stewart, J. P. Gaebler, T. E. Drake, and D. S. Jin, Evolution of the Normal State of a Strongly Interacting Fermi Gas from a Pseudogap Phase to a Molecular Bose Gas, Phys. Rev. Lett. 106, 060402 (2011). 